\title{
Renal outcomes in Asian patients receiving oral anticoagulants for non-valvular atrial fibrillation
}

\author{
Tayyab Salim Shahzada, Cosmos L Guo, Alex PW Lee *
}

\section{A B S T R A C T}

Introduction: Patients with non-valvular atrial fibrillation (NVAF) may be prescribed warfarin or a non-vitamin $\mathrm{K}$ oral anticoagulant (NOAC). There is increasing evidence that NOACs are superior to warfarin in terms of renal function preservation. This study aimed to compare renal outcomes in Chinese patients with NVAF between patients receiving NOACs and patients receiving warfarin.

Methods: In total, 600 Chinese patients with NVAF receiving oral anticoagulant therapy were retrospectively identified from an administrative database. The renal outcomes $(\geq 30 \%$ decline in estimated glomerular filtration rate [eGFR], doubling of serum creatinine, and kidney failure) were compared among four propensity-weighted treatment cohorts (warfarin, $\mathrm{n}=200$; rivaroxaban, $\mathrm{n}=200$; dabigatran, $\mathrm{n}=100$; and apixaban, $\mathrm{n}=100$ ).

Results: The mean follow-up period across all groups was $1000 \pm 436$ days. Compared with warfarin, the three NOACs (pooled for consideration as a single unit) had significantly lower risks of
95\% confidence interval $[\mathrm{CI}]=0.276-0.417)$ and doubling of serum creatinine $(\mathrm{HR}=0.550$; 95\% CI=0.387-0.782). Dabigatran and rivaroxaban users both had lower risks of $\geq 30 \%$ decline in eGFR (both $\mathrm{P}<0.001$ ) and doubling of serum creatinine (both $\mathrm{P}<0.05)$. Apixaban was only significantly associated with a lower risk of $\geq 30 \%$ decline in eGFR $(\mathrm{P}<0.001)$.

Conclusions: Compared with warfarin, NOACs may be associated with a significantly lower risk of decline in renal function among Chinese patients with NVAF.
Hong Kong Med J 2022;28:24-32
https://doi.org/10.12809/hkmj209201

\section{TS Shahzada}
CL Guo
APW Lee *, MD, FRCP
Department of Medicine and Therapeutics, The Chinese University of Hong Kong, Hong Kong

$\geq 30 \%$ decline in eGFR (hazard ratio $[\mathrm{HR}]=0.339$; * Corresponding author: alexpwlee@cuhk.edu.hk

\section{New knowledge added by this study}

- Decline in kidney function is common among Chinese patients who receive oral anticoagulant treatment for non-valvular atrial fibrillation.

- Warfarin usage is associated with significant long-term decline in renal function among patients treated for non-valvular atrial fibrillation.

- Compared with warfarin, non-vitamin K oral anticoagulant (NOAC) usage may be associated with a reduced risk of long-term decline in renal function among Chinese patients.

Implications for clinical practice or policy

- Patients receiving oral anticoagulants, especially warfarin, should undergo close renal function monitoring during the course of treatment.

- Considering that the decline in renal function may be more accelerated in warfarin users than in NOAC users, clinicians may consider preferential use of NOACs for anticoagulant therapy, especially in patients with existing renal impairment or risk factors for future decline in renal function.

- The inconsistencies of NOAC prescribing patterns with drug labelling in routine clinical practice should receive greater attention because dose reduction in the absence of a renal indication may reduce treatment effectiveness without providing a greater safety benefit.

\section{Introduction}

Various randomised controlled trials have demonstrated that non-vitamin $\mathrm{K}$ oral anticoagulants (NOACs), including factor Xa and direct thrombin inhibitors, are superior to warfarin, a vitamin K antagonist, in terms of efficacy and safety for preventing stroke and systemic thromboembolisms in patients with non-valvular atrial fibrillation
(NVAF). ${ }^{1-4}$ The superiority of NOACs compared with warfarin appears to be consistent across ethnic groups, including Asian populations. ${ }^{5}$ Furthermore, data from two sub-studies of the NOAC trials ${ }^{6,7}$ and a real-world cohort study ${ }^{8}$ suggested that NOACs may also be superior to warfarin in terms of maintaining and preserving renal function. A US-based cohort study demonstrated a lower 
risk of decline in renal function among patients receiving NOACs than among patients receiving warfarin. ${ }^{8}$ Moreover, findings from the ROCKET AF (Rivaroxaban Once-Daily, Oral, Direct Factor Xa Inhibition Compared With Vitamin K Antagonism for Prevention of Stroke and Embolism Trial in Atrial Fibrillation) and RE-LY (Randomized Evaluation of Long Term Anti-coagulation Therapy) trials revealed more rapid estimated glomerular filtration rate (eGFR) decline in patients receiving warfarin than in patients receiving rivaroxaban and dabigatran, respectively. ${ }^{6-8}$ Further studies have demonstrated that warfarin treatment may be associated with more rapid progression of chronic kidney disease and can cause acute kidney injury. ${ }^{8-10}$ This decline in renal function has been attributed to a phenomenon known as 'warfarin-related nephropathy', which is associated with vitamin $\mathrm{K}$ antagonism and excessive anticoagulation. ${ }^{8,9,11}$ In contrast, NOACs may offer renovascular protection through pharmacological mechanisms such as the inhibition of thrombin and factor $\mathrm{Xa} .{ }^{8,12,13}$

Differences in the pharmacological actions of warfarin and NOACs are reflected in the growing research that suggests NOACs are more effective than warfarin for preserving renal function. ${ }^{8,14}$ Considering that Asian warfarin users tended to have a lower time in therapeutic range (TTR) $)^{15,16}$ of the international normalised ratio (INR), which is associated with decline in renal function, ${ }^{6,14}$ the renal effects of NOACs compared with warfarin may differ from the effects in non-Asians. Dosage prescription patterns, such as the frequency of lowdose NOAC prescriptions, also vary between Asian and non-Asian populations ${ }^{14,17,18}$; this may also affect renal outcomes because the renal effects of NOACs appear to be dose-dependent. ${ }^{14,19}$ Furthermore, because of differences in NOAC-related bleeding risk between Asian and non-Asian populations, the renal protection effects of NOACs may also vary; major bleeding can cause decline in renal function. ${ }^{5,14,20,21}$ To our knowledge, there remain limited data comparing NOACs to warfarin in terms of decline in renal function among Asian patients. In this study, we sought to assess the renal outcomes of an ethnic Chinese patient population with NVAF who received NOACs (ie, apixaban, dabigatran, and rivaroxaban) compared with patients who received warfarin

\section{Methods}

\section{Study design}

This retrospective cohort study included four study groups: warfarin, apixaban, dabigatran, and rivaroxaban. Each NOAC was compared with warfarin.

\section{非瓣膜性心房顫動的亞洲患者接受口服抗凝血藥 治療的腎臟結局}

Tayyab Salim Shahzada、郭柳䤾、李沛威

引言：非瓣膜性心房顫動（NVAF）患者可獲處方華法林或非維他命K 口服抗凝血藥（NOAC）。越來越多證據表明NOAC比華法林更有效 保護腎臟功能。本研究旨在比較服用NOAC患者和服用華法林患者對 腎臟結局的影響。

方法: 從行政數據庫中追溯600名接受口服抗凝血藥治療的華籍NVAF 患者, 分為四個傾向加權治療隊列（華法林200例、利伐沙班200例

達比加群 100 例、阿哌沙班 100 例）比較腎臟功能（即估算的腎小球濾 過率eGFR下跌30\%或以上、血清肌酐加倍及腎衰竭）

結果：整體的平均隨訪時間為 $1000 \pm 436$ 天。與華法林組相比，三組 NOAC（合併為一個組別考慮）eGFR下跌 $30 \%$ 或以上風險（風險比 $0.339 ; 95 \%$ 置信區間=0.276-0.417）以及血清肌酐加倍的風險均較低 （風險比 $0.550 ； 95 \%$ 置信區間=0.387-0.782）。達比加群和利伐沙班 組eGFR下跌 $30 \%$ 或以上風險（兩者均 $\mathrm{P}<0.001$ ) 和血清肌酐加倍的風 險均明顯較低（兩者均 $\mathrm{P}<0.05$ ）。阿哌沙班僅與eGFR下跌 $30 \%$ 或以 上的風險較低顯著相關（ $\mathrm{P}<0.001 ）$

結論：與華法林相比, NOAC可能與華籍NVAF患者腎功能下降風險 顯著降低相關。

\section{Study population}

Data were extracted from patients' electronic medical records in the Prince of Wales Hospital of Hong Kong. In total, 2346 consecutive patients with a prescription of warfarin or one of the three NOACs (apixaban, rivaroxaban, and dabigatran) in our hospital were screened for eligibility for this analysis. Inclusion criteria were: first began to receive an oral anticoagulant between 1 January 2012 and 31 December 2016, NVAF, age $\geq 18$ years, minimum on-treatment duration of 3 months, and availability of laboratory data concerning serum creatinine at baseline and during follow-up. We excluded warfarin-experienced or NOAC-experienced patients to minimise confounding bias. ${ }^{8,22}$ Other exclusion criteria were previous kidney failure, valvular atrial fibrillation, ${ }^{23}$ and/or other indications for anticoagulation. A pre-study power analysis to detect a $10 \%$ difference in the incidence of $\geq 30 \%$ decline in eGFR between NOACs (pooled for consideration as a single unit) and warfarin revealed that the minimum sample size was 200 patients per arm (all NOACs vs warfarin). The sample size in each NOAC group was then matched to the approximate proportion of patients that were prescribed each of the three NOACs in actual clinical practice, in accordance with the preferences of local physicians. In our hospital during the study period, rivaroxaban was available earlier locally and was more frequently prescribed than the other two NOACs; apixaban 
was the last NOAC to receive local approval and therefore exhibited a lower rate of prescription at the time of the study. This paper adheres to the STROBE reporting guidelines for observational studies.

\section{Study endpoints}

We studied the three renal outcome endpoints: $\geq 30 \%$ decline in eGFR, doubling of serum creatinine, and kidney failure. Doubling of serum creatinine has been used as a surrogate endpoint when studying the progression of kidney disease in clinical trials. ${ }^{24}$ Based on the findings of clinical trials and metaanalyses, the National Kidney Foundation and US Food and Drug Administration proposed that with at least 2 to 3 years of follow-up, a $30 \%$ to $40 \%$ decline in eGFR may also be regarded as a surrogate end point; thus, it has been used as a renal endpoint in previous cohort analyses. ${ }^{8,24}$ Because doubling of serum creatinine and kidney failure occur late in kidney disease, $\geq 30 \%$ decline in eGFR serves as a more sensitive renal decline endpoint; this change is clinically significant regardless of a low follow-up or event rate. ${ }^{8,24}$ Kidney failure is defined as eGFR $<15 \mathrm{~mL} / \mathrm{min} / 1.73 \mathrm{~m}^{2}$, long-term kidney dialysis, or kidney transplantation. ${ }^{8,25}$ Efficacy outcomes were stroke (ischaemic or haemorrhagic) or systemic embolism (SE). Based on the initial dose prescribed, the prevalence of dose reduction for each NOAC (apixaban $2.5 \mathrm{mg}$ twice daily, dabigatran $75 \mathrm{mg}$ twice daily, and rivaroxaban $15 \mathrm{mg}$ once daily) without a renal indication (eGFR $<30 \mathrm{~mL} / \mathrm{min}$ for apixaban and dabigatran; eGFR $<50 \mathrm{~mL} / \mathrm{min}$ for rivaroxaban) was assessed. ${ }^{26}$

Using the treatment initiation date as our index date, we retrieved the pretreatment creatinine value nearest to the index date as the baseline creatinine; we used this value to calculate the baseline eGFR by means of the Chronic Kidney Disease Epidemiology Collaboration equation., ${ }^{8,27}$ Hospital electronic records were used to identify co-morbidities and specific drug prescriptions within 3 months prior to the index date. Baseline HAS-BLED and $\mathrm{CHA}_{2} \mathrm{DS}_{2}$-VASc scores were also recorded. The TTR of patients in the warfarin cohort was calculated as the number of INRs in therapeutic range (INR=2-3) divided by the total INRs recorded for each patient during the analysed period. ${ }^{28,29}$ Patients were followed up until the end of treatment, death, or when any efficacy or renal endpoint(s) were reached.

\section{Statistical analysis}

For minimisation of potential confounding, we used inverse probability of treatment weighting (IPTW) to balance identified covariates. Generalised boosted models were used to estimate propensity scores and weights for optimal balance across treatment groups. ${ }^{31,32}$ Weights were obtained to gather estimates representing the mean effects of treatment among treated groups. ${ }^{8,14}$ Baseline characteristics (eg, patient baseline medications and pre-existing co-morbidities which may affect outcomes) were included in our model (online supplementary Table). ${ }^{8,14}$ Both $\mathrm{CHA}_{2} \mathrm{DS}_{2}$-VASc and HAS-BLED scores were not included in the model because they are composite scores derived from other covariates. ${ }^{14}$ The absolute standardised mean difference was calculated for each NOAC versus warfarin to ensure that the cohorts were sufficiently balanced before comparison of each NOAC to warfarin. An absolute standardised mean difference of $<0.2$ is considered balanced for each baseline covariate when comparing each NOAC to warfarin. ${ }^{8,31}$ Fisher's exact test was used to compare the frequencies of dose reduction without renal indication among NOACs.

Because of some extremely high or low weights in our weighted population, we truncated weights at the 1st and 99th percentiles before conducting weighted analysis. ${ }^{8,33}$ We calculated hazard ratios using weighted Cox proportional hazards regression, then generated weighted Kaplan-Meier curves that compared each NOAC to warfarin. Cumulative incidences for the Kaplan-Meier curves were presented as mean percentage incidences with $95 \%$ confidence intervals. A P value of $<0.05$ was considered statistically significant. Predefined subgroup analysis was performed for factors potentially associated with renal outcome, including age ( $\geq 75$ or $<75$ years); sex; and baseline diabetes mellitus, heart failure, and eGFR $\left(\geq 60 \mathrm{~mL} / \mathrm{min} / 1.73 \mathrm{~m}^{2}\right.$ or $<60 \mathrm{~mL} / \mathrm{min} / 1.73 \mathrm{~m}^{2}$ ).

\section{Results \\ Cohort characteristics}

We identified 600 patients with NVAF who were receiving oral anticoagulants: 200, 100, 100, and 200 patients were receiving warfarin, apixaban, dabigatran, and rivaroxaban, respectively. After IPTW, all identified baseline characteristics were balanced between warfarin and each NOAC group (Table 1). The mean follow-up duration of the overall study cohort was $1000 \pm 436$ days. The mean follow-up durations for each NOAC group were as follows: apixaban $(790 \pm 345$ days $)$, dabigatran (1187 \pm 322 days), and rivaroxaban (999 \pm 430 days); the median follow-up durations were 806, 1416, and 1074 days, respectively. The mean TTR of the warfarin cohort was $44.3 \%$. The frequencies of dose reduction without a renal indication for apixaban, rivaroxaban and dabigatran were $46.9 \%, 35.7 \%$ and $2.0 \%$, respectively $(\mathrm{P}<0.001$ for dabigatran vs both apixaban and rivaroxaban). 
TABLE I. Baseline characteristics after inverse probability of treatment weighting*

\begin{tabular}{|c|c|c|c|c|c|c|c|}
\hline & \multirow{2}{*}{$\begin{array}{l}\text { Warfarin } \\
(n=200)\end{array}$} & \multirow{2}{*}{$\begin{array}{l}\text { Apixaban } \\
(n=100)\end{array}$} & \multirow{2}{*}{$\begin{array}{l}\text { Dabigatran } \\
(n=100)\end{array}$} & \multirow{2}{*}{$\begin{array}{l}\text { Rivaroxaban } \\
(n=200)\end{array}$} & \multicolumn{3}{|c|}{ Absolute SMD } \\
\hline & & & & & $\begin{array}{l}\text { Warfarin vs } \\
\text { apixaban }\end{array}$ & $\begin{array}{l}\text { Warfarin vs } \\
\text { dabigatran }\end{array}$ & $\begin{array}{l}\text { Warfarin vs } \\
\text { rivaroxaban }\end{array}$ \\
\hline Age at prescription & $76.2 \pm 9.0$ & $75.8 \pm 10.3$ & $77.8 \pm 7.3$ & $76.6 \pm 8.4$ & 0.044 & 0.196 & 0.045 \\
\hline Female sex & 46.8 & 55.7 & 51.0 & 49.8 & 0.177 & 0.083 & 0.058 \\
\hline HAS-BLED score & $2.8 \pm 1.1$ & $2.6 \pm 1.1$ & $2.9 \pm 0.9$ & $2.6 \pm 1.0$ & 0.165 & 0.102 & 0.167 \\
\hline $\mathrm{CHA}_{2} \mathrm{DS}_{2}$-VASc score & $4.1 \pm 1.7$ & $4.1 \pm 1.7$ & $4.2 \pm 1.7$ & $4.2 \pm 1.6$ & 0.004 & 0.066 & 0.050 \\
\hline Heart failure & 24.1 & 20.6 & 20.1 & 22.9 & 0.084 & 0.097 & 0.028 \\
\hline Hypertension & 76.5 & 74.5 & 76.5 & 79.4 & 0.046 & 0.001 & 0.072 \\
\hline Diabetes mellitus & 37.0 & 28.0 & 37.9 & 34.9 & 0.192 & 0.019 & 0.044 \\
\hline $\begin{array}{l}\text { History of ischaemic stroke / TIA / } \\
\text { systemic thromboembolism }\end{array}$ & 27.7 & 34.7 & 23.2 & 30.2 & 0.151 & 0.104 & 0.055 \\
\hline History of bleeding & 4.8 & 7.7 & 5.9 & 3.5 & 0.122 & 0.049 & 0.064 \\
\hline Prior myocardial infarction & 17.1 & 20.5 & 16.8 & 17.0 & 0.087 & 0.007 & 0.003 \\
\hline History of cancer & 9.3 & 10.7 & 9.6 & 8.8 & 0.048 & 0.013 & 0.016 \\
\hline Antiplatelet therapy & 59.1 & 51.3 & 55.8 & 55.9 & 0.156 & 0.066 & 0.065 \\
\hline Other NSAID & 4.0 & 5.6 & 5.9 & 1.6 & 0.076 & 0.088 & 0.146 \\
\hline Statin therapy & 48.6 & 49.5 & 45.4 & 51.0 & 0.019 & 0.064 & 0.048 \\
\hline Diuretic therapy & 33.1 & 29.8 & 33.4 & 27.8 & 0.070 & 0.008 & 0.114 \\
\hline ACEi / ARB / ARNi / MRA & 52.6 & 49.9 & 57.0 & 54.2 & 0.053 & 0.090 & 0.032 \\
\hline eGFR, mL/min/1.73 m² & $63.7 \pm 18.8$ & $64.1 \pm 18.5$ & $64.7 \pm 16.2$ & $64.6 \pm 18.7$ & 0.021 & 0.058 & 0.047 \\
\hline
\end{tabular}

Abbreviations: $\mathrm{ACEi}=$ angiotensin-converting enzyme inhibitors; $\mathrm{ARB}=$ angiotensin II receptor blocker; $\mathrm{ARNi}=$ angiotensin receptor-neprilysin inhibitors; $\mathrm{CHA}_{2} \mathrm{DS}_{2}-\mathrm{VASC}=$ congestive heart failure, hypertension, age $\geq 75$ years, age 65 to 74 years, stroke/transient ischaemic attack/thromboembolism, vascular disease, diabetes mellitus, sex (female); eGFR = estimated glomerular filtration rate; HAS-BLED = hypertension, abnormal renal or liver function, stroke, bleeding, labile international normalised ratio, elderly (age >65 years), drugs (eg, antiplatelet), or alcohol; MRA = mineralocorticoid receptor antagonists; NSAID = non-steroidal anti-inflammatory drug; SMD = standardised mean difference; TIA = transient ischaemic attack

Data are shown as mean \pm standard deviation or $\%$

\section{Renal and efficacy outcomes}

When the three NOACs were pooled for consideration as a single unit and compared with warfarin, NOAC users exhibited lower risks of $\geq 30 \%$ decline in eGFR (hazard ratio $[\mathrm{HR}]=0.339$; 95\% confidence interval $[\mathrm{CI}]=0.276-0.417 ; \mathrm{P}<0.001$ ) and doubling of serum creatinine $(\mathrm{HR}=0.550$; 95\% $\quad \mathrm{CI}=0.387-0.782 ; \quad \mathrm{P}<0.001) . \quad$ Individual comparisons of each NOAC to warfarin (Table 2) revealed that dabigatran and rivaroxaban users both had lower risks of $\geq 30 \%$ decline in eGFR (both $\mathrm{P}<0.001$ ) and doubling of serum creatinine (both $\mathrm{P}<0.05$ ). However, apixaban users only had a lower risk of $\geq 30 \%$ decline in eGFR $(P<0.001)$. Despite trends suggestive of lower kidney failure risk in patients receiving dabigatran or rivaroxaban, the overall use of NOACs was not significantly associated with lower kidney failure risk, compared with the use of warfarin. Figure 1 shows the weighted Kaplan-Meier curves for the renal endpoints. For the efficacy outcome, dabigatran was associated with a lower incidence of stroke/SE $(\mathrm{HR}=0.151$; 95\% $\mathrm{CI}=0.054-0.423) ; \mathrm{P}<0.001$ vs warfarin), whereas the use of apixaban or rivaroxaban was not significantly associated with a lower incidence of stroke/SE, compared with the use of warfarin (Fig 2).
TABLE 2. Hazard ratios with $95 \%$ confidence intervals $(95 \% \mathrm{Cl})$

\begin{tabular}{lcc}
\hline & Hazard ratio $(95 \%$ Cl) & P value \\
\hline $\begin{array}{l}\text { z30\% Decline in estimated glomerular } \\
\text { filtration rate }\end{array}$ & \\
\hline Apixaban & $0.552(0.418-0.730)$ & $<0.001$ \\
\hline Dabigatran & $0.203(0.144-0.285)$ & $<0.001$ \\
\hline Rivaroxaban & $0.343(0.260-0.451)$ & $<0.001$ \\
\hline Warfarin & Reference & Reference \\
\hline Doubling of serum creatinine & & \\
\hline Apixaban & $0.914(0.566-1.474)$ & 0.711 \\
\hline Dabigatran & $0.525(0.333-0.829)$ & 0.006 \\
\hline Rivaroxaban & $0.311(0.180-0.536)$ & $<0.001$ \\
\hline Warfarin & Reference & Reference \\
\hline Kidney failure & $1.451(0.804-2.619)$ & \\
\hline Apixaban & $0.544(0.268-1.105)$ & 0.217 \\
\hline Dabigatran & $0.544(0.273-1.083)$ & 0.092 \\
\hline Rivaroxaban & Reference & Reference \\
\hline Warfarin & & \\
\hline All stroke (ischaemic or haemorrhagic) & & \\
\hline or systemic embolism & $0.803(0.424-1.522)$ & 0.501 \\
\hline Apixaban & $0.151(0.054-0.423)$ & $<0.001$ \\
\hline Dabigatran & $0.613(0.340-1.104)$ & 0.103 \\
\hline Rivaroxaban & Reference & Reference \\
\hline Warfarin & & \\
\hline & & \\
\hline
\end{tabular}




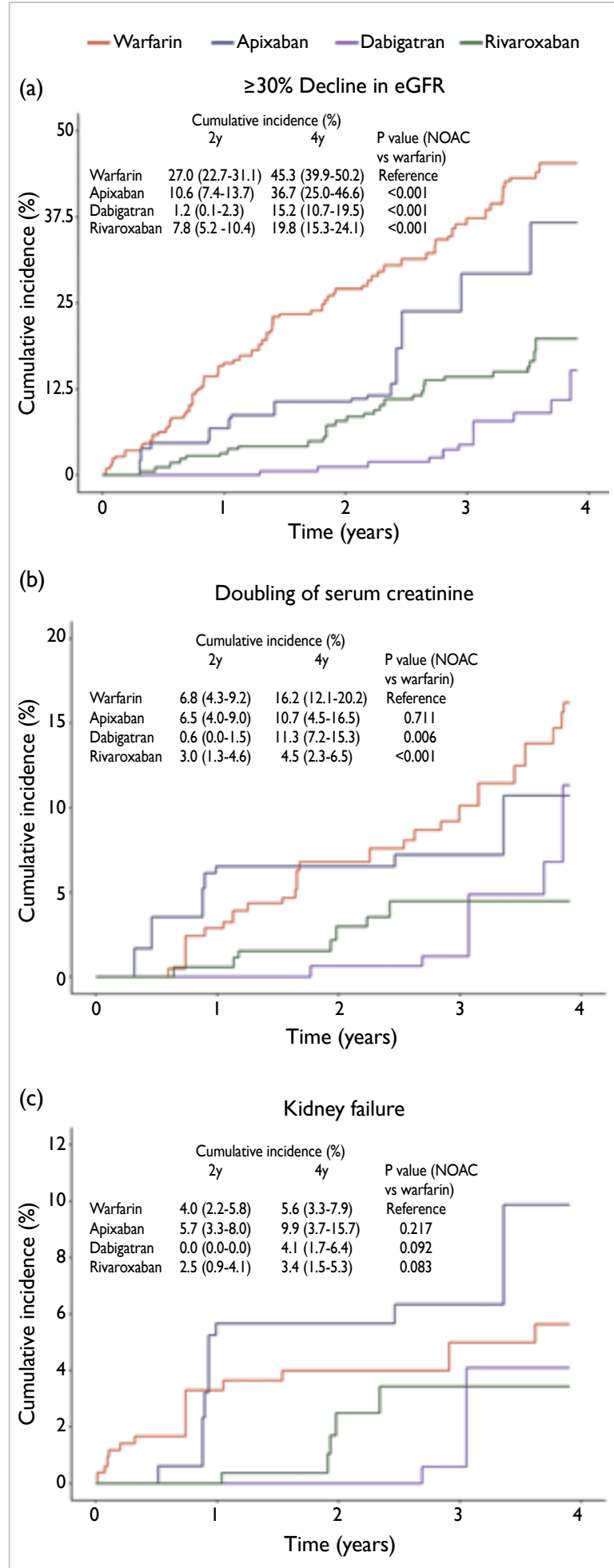

FIG I. Cumulative incidences of renal endpoints in patients receiving warfarin and non-vitamin $\mathrm{K}$ oral anticoagulants (NOACs). (a-c) Weighted Kaplan-Meier cumulative incidences (\%) and $95 \%$ confidence intervals at 2 years $(2 y)$ and 4 years (4y) using inverse probability treatment weighting. $P$ values when comparing curves for each NOAC to warfarin are shown. Dabigatran and rivaroxaban were both associated with lower risk of $\geq 30 \%$ decline in estimated glomerular filtration rate (eGFR) and doubling of serum creatinine; apixaban was associated with lower risk of $\geq 30 \%$ decline in eGFR

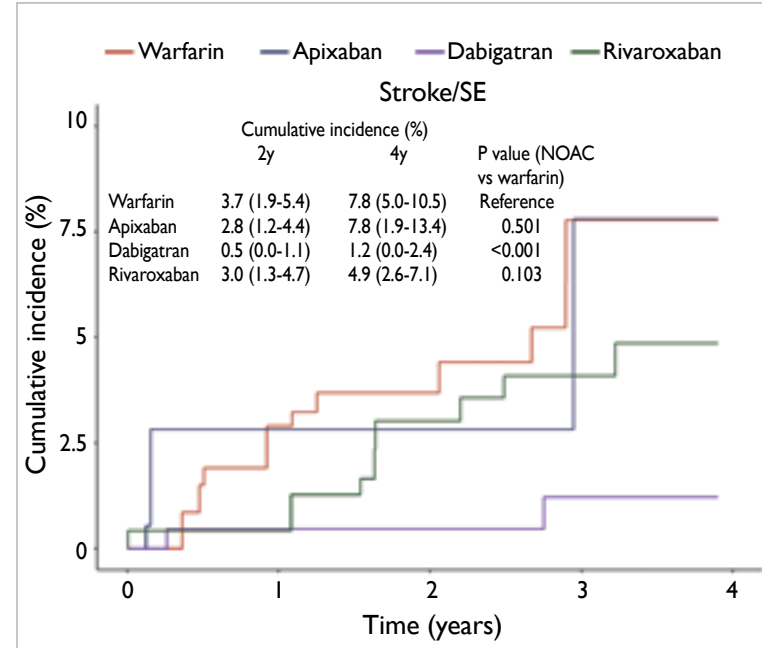

FIG 2. Cumulative incidences of stroke/systemic embolism (SE) in patients receiving warfarin and non-vitamin $\mathrm{K}$ oral anticoagulants (NOACs). Weighted Kaplan-Meier cumulative incidences (\%) and $95 \%$ confidence intervals at 2 years $(2 y)$ and 4 years (4y) using inverse probability treatment weighting. $P$ values when comparing curves for each NOAC to warfarin are shown. Dabigatran was associated with a lower risk of stroke/SE compared with warfarin

\section{Subgroup analysis}

Analysis of the main renal endpoint, $\geq 30 \%$ decline in eGFR, consistently favoured the use of dabigatran or rivaroxaban, compared with warfarin, across all subgroups (Fig 3). However, the use of apixaban was not associated with a reduced risk of $\geq 30 \%$ decline in eGFR in three subgroups: men $(\mathrm{P}=0.057)$, patients with heart failure $(\mathrm{P}=0.835)$, and patients without diabetes mellitus $(\mathrm{P}=0.090)$.

\section{Discussion}

\section{Summary and potential mechanisms}

Our cohort study provides important insights into the long-term renal impacts of NOACs versus warfarin in an ethnic Chinese population. Decline in renal function was evident among both warfarin and NOAC users in our cohort. However, the use of NOACs was generally associated with better long-term renal outcomes, compared with the use of warfarin, among Chinese patients. The general superiority of NOACs compared with warfarin was most evident for the $\geq 30 \%$ decline in eGFR surrogate endpoint. The use of dabigatran or rivaroxaban was associated with lower risks of $\geq 30 \%$ decline in eGFR and doubling of creatinine in the overall population and across predefined demographic and clinical subgroups; in contrast, the use of apixaban was not associated with a lower risk of doubling of serum creatinine in the overall population, nor was it associated with a lower risk of $\geq 30 \%$ decline in eGFR among several subgroups (men, patients without diabetes mellitus, and patients with heart failure). 
Pharmacological mechanisms may explain our findings concerning NOAC superiority. Because warfarin is a vitamin $\mathrm{K}$ antagonist, it has inhibitory effects on matrix gamma-carboxyglutamic acid, a vitamin $\mathrm{K}$-dependent protein which normally protects against vascular calcification; thus, warfarin administration potentially stimulates and accelerates the calcification of renal vascular tissue, which promotes nephropathy. ${ }^{8,34,35}$ The mechanism of warfarin-related nephropathy has various contributing factors, such as the occurrence of glomerular haemorrhage and subsequent tubular injury because of red blood cell casts and haemrelated free radical injury. ${ }^{10,36,37}$ Alternatively, NOACs may offer renovascular protection through distinct mechanisms such as the inhibition of thrombin and factor $\mathrm{Xa}{ }^{8,12,13}$

\section{Comparison with existing literature}

Our data are generally consistent with previous studies concerning the renal outcomes of NOACs versus warfarin. A study by Yao et $\mathrm{al}^{8}$ regarding the renal outcomes of NOACs showed that dabigatran was associated with a lower risk of $\geq 30 \%$ decline in eGFR, while rivaroxaban was associated with lower risks of $\geq 30 \%$ decline in eGFR and doubling of serum creatinine. Analysis of the RE-LY and ROCKET AF trials similarly showed more rapid decline in eGFR among warfarin users, compared with dabigatran and rivaroxaban users. ${ }^{6-8}$ Hernandez et $\mathrm{al}^{38}$ demonstrated rivaroxaban superiority for adverse renal events, compared with warfarin, in patients with NVAF who had diabetes mellitus. However, our results for apixaban were inconsistent with the findings of the ARISTOTLE trial, which did not show significant apixaban superiority in terms of renal function preservation; the analysis showed similar but slightly greater decline in eGFR among apixaban users, compared with warfarin users. ${ }^{8,39}$ Yao et $\mathrm{al}^{8}$ also showed no clear benefits for apixaban, compared with warfarin, in terms of renal protection. Nonetheless, a study in Taiwan by Chan et $\mathrm{al}^{14}$ showed that, compared with warfarin, all three NOACs were associated with lower risk for acute kidney injury in both chronic kidney disease-free and chronic kidney disease cohorts.

The differences between our findings and the results of previous studies-especially with respect to apixaban in our cohort versus the ARISTOTLE subanalysis ${ }^{39}$-may have several explanations. As mentioned by Chan et al, ${ }^{14}$ Asian populations tended to have lower TTR with warfarin usage, compared with non-Asians ${ }^{15,16}$; our warfarin cohort had a mean TTR of $44.3 \%$, which was considerably lower than findings in non-Asian populations. ${ }^{15,16}$ Combined with findings that renal deterioration is greater when

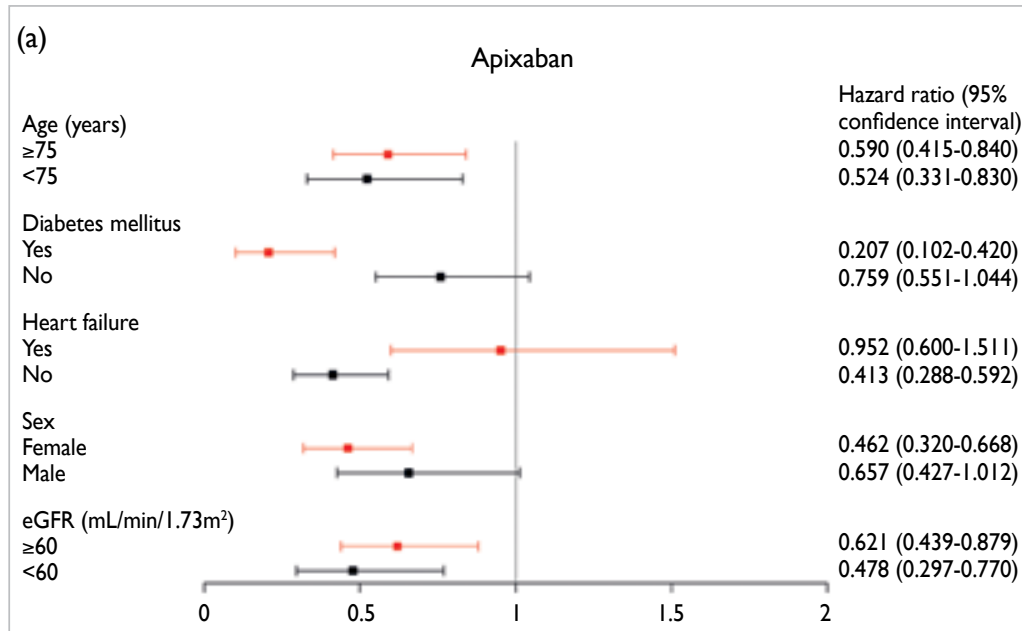

(b)

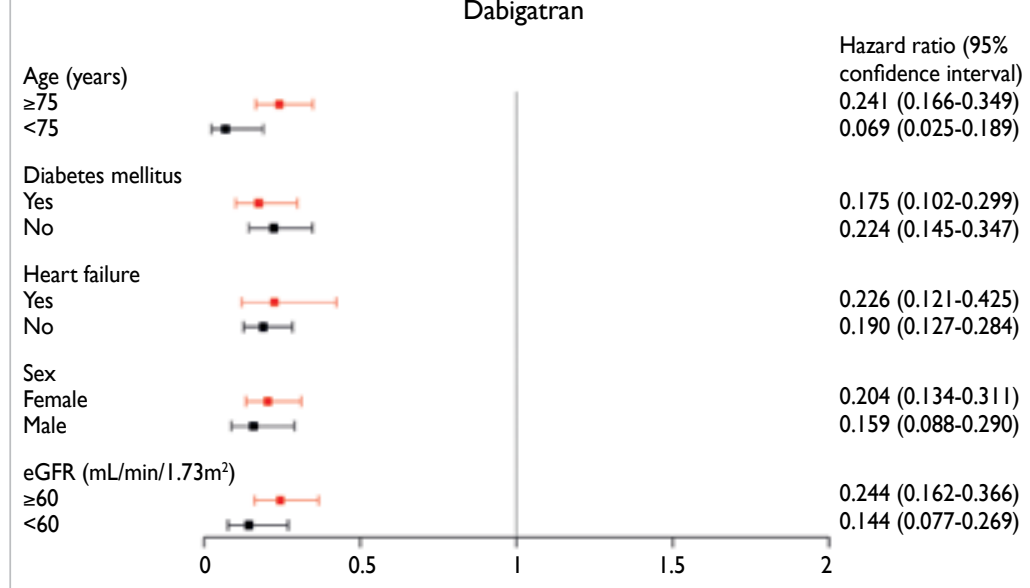

(c)

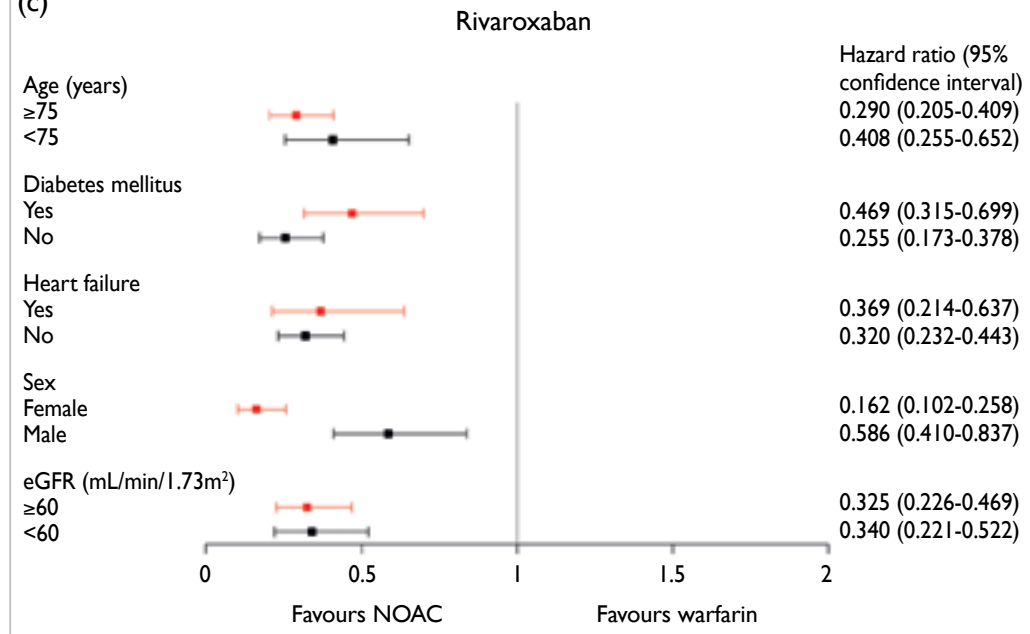

FIG 3. Subgroup analysis for $\geq 30 \%$ decline in eGFR. (a-c) Hazard ratios for predefined subgroups comparing each non-vitamin $\mathrm{K}$ oral anticoagulant to warfarin. Dabigatran and rivaroxaban were associated with lower risk of $\geq 30 \%$ decline in estimated glomerular filtration rate (eGFR) in all subgroups; apixaban was associated with lower risk of $\geq 30 \%$ decline in eGFR in most subgroups (with exceptions of patients without diabetes mellitus, patients with heart failure, and male patients) 
warfarin is poorly controlled-especially with INR levels above the target range, as demonstrated in the RE-LY trial ${ }^{6}$-indicates that Asian populations, such as the Chinese, may have an elevated risk of warfarin-related nephropathy. ${ }^{14}$ Because Asian patients may be more susceptible to renal decline associated with warfarin use, apixaban may appear superior to warfarin in Asian populations, although this superiority may not persist in non-Asian populations. ${ }^{8}$ Additional apixaban superiority in Asian populations, as discussed by Chan et $\mathrm{al},{ }^{14}$ may also be explained by the superior efficacy and safety of NOACs in Asians, compared with non-Asians. 5,21 Because major bleeding can be associated with renal function deterioration, the greater efficacy and safety of NOACs in Asians may facilitate renal risk reduction in such populations ${ }^{5,14,21}$ Notably, there was a high prevalence of non-guideline dose reduction without a renal indication ${ }^{26}$ in the apixaban group, compared with other NOACs, in our study; this dose reduction has been associated with worse stroke prevention effectiveness and provides no safety benefit. ${ }^{40}$ Nonetheless, apixaban was not significantly associated with risk reduction of the other two renal outcomes in our study, compared with warfarin. This may suggest uncertainty concerning its renal risk reduction superiority compared with warfarin. Overall, such inconsistencies across studies indicate the need for additional research; they may also reflect insufficient statistical power in our study to generate more robust conclusions.

The aforementioned findings concerning greater risk of warfarin-related nephropathy and possible lower risk of renal decline with NOAC usage in Asian populations are also potentially reflected in the comparatively lower HRs for renal endpoints in our study, compared with the USbased cohort reported by Yao et al. ${ }^{8}$ When the NOACs were pooled (for consideration as a single unit) and compared with warfarin, HRs for $\geq 30 \%$ decline in eGFR and doubling of serum creatinine were both lower in our population, compared with the pooled results described by Yao et $\mathrm{al}^{8}(\mathrm{HR}=0.77$; 95\% $\mathrm{CI}=0.66-0.89$ and $\mathrm{HR}=0.62 ; 95 \% \mathrm{CI}=0.40-0.95$ ).

\section{Strengths and limitations}

Notable strengths of our study were its long study period and subsequent long mean follow-up duration. The longer follow-up duration, compared with previous cohort studies, indicates that previous findings concerning NOAC superiority for renal outcomes also persist during longer follow-up periods. Furthermore, our database comprised each patient's complete laboratory data; this allowed accurate recording of each renal outcome through serum creatinine and eGFR values, thus enhancing the consistency and preciseness of renal measurement across all patients. We minimised potential confounding by only including patients who were first-time users of oral anticoagulants; this enabled us to balance numerous important baseline characteristics.

Regarding limitations, although we utilised IPTW to balance baseline covariates, confounding bias may have persisted in the study. ${ }^{8,14}$ Nonetheless, we achieved balance concerning the most important identified baseline covariates that may impact renal function across treatment groups. Moreover, although the smaller number of events may have limited the statistical power with respect to the less sensitive endpoint of kidney failure, by including $\geq 30 \%$ decline in eGFR as a sensitive renal outcome, we were able to sufficiently assess early renal decline. Smaller declines in renal function (eg, $\geq 30 \%$ decline in eGFR) serve as valuable and sensitive indicators of renal decline ${ }^{8}$ that has been regarded as a useful surrogate endpoint for progression to kidney failure ${ }^{24}$; it is also reportedly associated with risks of end-stage renal disease and mortality ${ }^{41}$ Frequency of testing, as mentioned in Yao et $\mathrm{al}^{8}{ }^{8}$ may also affect results; the inclusion of patients with more follow-up creatinine tests leads to greater sensitivity concerning outcome incidence, compared with patients who underwent fewer tests. To minimise the potential impact of this sensitivity on the renal endpoints, we only included patients for whom creatinine tests were available throughout the entire follow-up period; this was possible because all patients were treated in a single centre.

Overall, the general consistency of our results with the findings of previous cohort studies, as well as the findings of the RE-LY and ROCKET AF trials, enhances the reliability and robustness of our results. ${ }^{6-8,14}$ Nonetheless, further studies are needed to identify consistencies among the existing discrepancies, especially concerning apixaban. Greater certainty regarding renal outcomes of all NOACs is also important because one previous meta-analysis of various randomised controlled trials concluded that the risk of kidney failure associated with NOACs was similar to the risk associated with other anticoagulants. ${ }^{42}$ Finally, although this study only involved Hong Kong Chinese patients, whose responses to NOACs and warfarin may differ from the responses of their non-Asian counterparts, the consistency of the results with findings from studies in other regions suggests widespread applicability of the findings.

\section{Clinical implications}

In patients with NVAF who are receiving oral anticoagulants, gradual renal impairment is associated with worse clinical outcomes. . $^{39,43}$ Our results suggested that patients receiving oral anticoagulant therapy, particularly warfarin, should undergo close renal function monitoring. Decline 
in renal function during anticoagulant therapy may be less likely to occur when receiving NOACs than when receiving warfarin. The NOAC efficacy findings in this study were generally consistent with previously reported data in terms of stroke/SE prevention non-inferiority or superiority, compared with warfarin. ${ }^{44-47}$ In particular, the superior efficacy of dabigatran compared with warfarin, in this local study population is reassuring. The inconsistencies of NOAC prescribing patterns with drug labelling in routine clinical practice, particularly regarding apixaban, should receive greater attention, because dose reduction in the absence of a renal indication has been associated with worse effectiveness and no safety benefit in apixaban-treated patients with normal or mildly impaired renal function. ${ }^{40}$

\section{Conclusions}

Compared with warfarin, NOAC treatment may be associated with a lower risk of renal decline in Chinese populations; this should be considered by clinicians during the selection of anticoagulant treatment. Further studies are needed in Asian populations (eg, Chinese) to better understand the renal superiority or inferiority of NOACs compared with warfarin. Besides, NOAC-to-NOAC comparisons are needed to inform treatment selection. Additional research is needed in specific populations, such as patients with diabetes mellitus or heart failure, to better understand the impacts of baseline co-morbidities on renal risk reduction related to the use of NOACs, compared with the use of warfarin. Large-scale studies should also investigate how dosage patterns may influence renal outcomes.

\section{Author contributions}

Concept or design: APW Lee.

Acquisition of data: All authors.

Analysis or interpretation of data: All authors.

Drafting of the manuscript: All authors.

Critical revision of the manuscript for important intellectual content: APW Lee.

All authors had full access to the data, contributed to the study, approved the final version for publication, and take responsibility for its accuracy and integrity.

\section{Conflicts of interest}

APW Lee has received research grants from Bayer, Pfizer, and Boehringer Ingelheim.

\section{Funding/support}

This work was funded by the Hong Kong SAR Government Health and Medical Research Fund (05160976). The funder had no role in study design, data collection/analysis/ interpretation, or manuscript preparation.

\section{Ethics approval}

The study was approved by The Joint Chinese University of
Hong Kong-New Territories East Cluster Clinical Research Ethics Committee (Ref CREC 2019.405). Informed consent was waived because of the retrospective nature of this study.

\section{References}

1. Hart RG, Pearce LA, Aguilar MI. Meta-analysis: antithrombotic therapy to prevent stroke in patients who have nonvalvular atrial fibrillation. Ann Intern Med 2007;146:857-67.

2. Connolly SJ, Ezekowitz MD, Yusuf S, et al. Dabigatran versus warfarin in patients with atrial fibrillation. $\mathrm{N}$ Engl J Med 2009;361:1139-51.

3. Patel MR, Mahaffey KW, Garg J, et al. Rivaroxaban versus warfarin in nonvalvular atrial fibrillation. N Engl J Med 2011;365:883-91.

4. Granger CB, Alexander JH, McMurray JJ, et al. Apixaban versus warfarin in patients with atrial fibrillation. $\mathrm{N}$ Engl J Med 2011;365:981-92.

5. Lip GY, Wang KL, Chiang CE. Non-vitamin K antagonist oral anticoagulants (NOACs) for stroke prevention in Asian patients with atrial fibrillation: time for a reappraisal. Int J Cardiol 2015;180:246-54.

6. Böhm M, Ezekowitz MD, Connolly SJ, et al. Changes in renal function in patients with atrial fibrillation: an analysis from the RE-LY trial. J Am Coll Cardiol 2015;65:2481-93.

7. Fordyce CB, Hellkamp AS, Lokhnygina Y, et al. Ontreatment outcomes in patients with worsening renal function with rivaroxaban compared with warfarin: Insights from ROCKET AF. Circulation 2016;134:37-47.

8. Yao X, Tangri N, Gersh BJ, et al. Renal outcomes in anticoagulated patients with atrial fibrillation. J Am Coll Cardiol 2017;70:2621-32.

9. Brodsky SV, Nadasdy $\mathrm{T}$, Rovin $\mathrm{BH}$, et al. Warfarinrelated nephropathy occurs in patients with and without chronic kidney disease and is associated with an increased mortality rate. Kidney Int 2011;80:181-9.

10. Brodsky SV, Satoskar A, Chen J, et al. Acute kidney injury during warfarin therapy associated with obstructive tubular red blood cell casts: a report of 9 cases. Am J Kidney Dis 2009;54:1121-6.

11. Brodsky SV, Collins M, Park E, et al. Warfarin therapy that results in an international normalization ratio above the therapeutic range is associated with accelerated progression of chronic kidney disease. Nephron Clin Pract 2010;115:142-6.

12. Sparkenbaugh EM, Chantrathammachart P, Mickelson J, et al. Differential contribution of FXa and thrombin to vascular inflammation in a mouse model of sickle cell disease. Blood 2014;123:1747-56.

13. Lee IO, Kratz MT, Schirmer SH, Baumhäkel M, Böhm M. The effects of direct thrombin inhibition with dabigatran on plaque formation and endothelial function in apolipoprotein E-deficient mice. J Pharmacol Exp Ther 2012;343:253-7.

14. Chan YH, Yeh YH, Hsieh MY, et al. The risk of acute kidney injury in Asians treated with apixaban, rivaroxaban, dabigatran, or warfarin for non-valvular atrial fibrillation: a nationwide cohort study in Taiwan. Int J Cardiol 2018;265:83-9.

15. Wallentin L, Yusuf S, Ezekowitz MD, et al. Efficacy and safety of dabigatran compared with warfarin at different levels of international normalised ratio control for stroke prevention in atrial fibrillation: an analysis of the RE-LY 
trial. Lancet 2010;376:975-83.

16. Singer DE, Hellkamp AS, Piccini JP, et al. Impact of global geographic region on time in therapeutic range on warfarin anticoagulant therapy: data from the ROCKET AF clinical trial. J Am Heart Assoc 2013;2:e000067.

17. Nielsen PB, Skjøth F, Søgaard M, Kjældgaard JN, Lip GY, Larsen TB. Effectiveness and safety of reduced dose nonVitamin $\mathrm{K}$ antagonist oral anticoagulants and warfarin in patients with atrial fibrillation: propensity weighted nationwide cohort study. BMJ 2017;356:j510.

18. Hori M, Matsumoto M, Tanahashi N, et al. Rivaroxaban vs. warfarin in Japanese patients with atrial fibrillation-the J-ROCKET AF study. Circ J 2012;76:2104-11.

19. Ryan M, Ware K, Qamri Z, et al. Warfarin-related nephropathy is the tip of the iceberg: direct thrombin inhibitor dabigatran induces glomerular hemorrhage with acute kidney injury in rats. Nephrol Dial Transplant 2014;29:2228-34

20. Makris K, Spanou L. Acute kidney injury: definition, pathophysiology and clinical phenotypes. Clin Biochem Rev 2016;37:85-98.

21. Wang KL, Lip GY, Lin SJ, Chiang CE. Non-vitamin K antagonist oral anticoagulants for stroke prevention in Asian patients with nonvalvular atrial fibrillation: metaanalysis. Stroke 2015;46:2555-61.

22. Ray WA. Evaluating medication effects outside of clinical trials: new-user designs. Am J Epidemiol 2003;158:915-20.

23. January CT, Wann LS, Calkins H, et al. 2019 AHA/ACC/ HRS focused update of the 2014 AHA/ACC/HRS guideline for the management of patients with atrial fibrillation: a report of the American College of Cardiology/American Heart Association Task Force on Clinical Practice Guidelines and the Heart Rhythm Society. J Am Coll Cardiol 2019;74:104-32.

24. Levey AS, Inker LA, Matsushita K, et al. GFR decline as an end point for clinical trials in CKD: a scientific workshop sponsored by the national kidney foundation and the US Food and Drug Administration. Am J Kidney Dis 2014;64:821-35.

25. Levin A, Stevens PE, Bilous RW, et al. Kidney disease: improving global outcomes (KDIGO) CKD work group. KDIGO 2012 clinical practice guideline for the evaluation and management of chronic kidney disease. Kidney Int Suppl 2013;3:1-150.

26. Lau YC, Proietti M, Guiducci E, Blann AD, Lip GY. Atria fibrillation and thromboembolism in patients with chronic kidney disease. J Am Coll Cardiol 2016;68:1452-64.

27. Levey AS, Inker LA, Coresh J. GFR estimation: from physiology to public health. Am J Kidney Dis 2014;63:820 34.

28. Lam AS, Lee IM, Mak SK, Yan BP, Lee VW. Warfarin control in Hong Kong clinical practice: a single-centre observational study. Hong Kong Med J 2020;26:294-303.

29. Schmitt L, Speckman J, Ansell J. Quality assessment of anticoagulation dose management: comparative evaluation of measures of time-in-therapeutic range. J Thromb Thrombolysis 2003;15:213-6.

30. Larsen TB, Skjøth F, Nielsen PB, Kjældgaard JN, Lip GY. Comparative effectiveness and safety of non-vitamin $\mathrm{K}$ antagonist oral anticoagulants and warfarin in patients with atrial fibrillation: propensity weighted nationwide cohort study. BMJ 2016;353:i3189.

31. McCaffrey DF, Griffin BA, Almirall D, Slaughter ME, Ramchand R, Burgette LF. A tutorial on propensity score estimation for multiple treatments using generalized boosted models. Stat Med 2013;32:3388-414.

32. Austin PC. An introduction to propensity score methods for reducing the effects of confounding in observational studies. Multivariate Behav Res 2011;46:399-424.

33. Austin PC, Stuart EA. Moving towards best practice when using inverse probability of treatment weighting (IPTW) using the propensity score to estimate causal treatment effects in observational studies. Stat Med 2015;34:366179.

34. Chatrou ML, Winckers K, Hackeng TM, Reutelingsperger CP, Schurgers LJ. Vascular calcification: the price to pay for anticoagulation therapy with vitamin K-antagonists. Blood Rev 2012;26:155-66.

35. Schurgers LJ, Joosen IA, Laufer EM, et al. Vitamin Kantagonists accelerate atherosclerotic calcification and induce a vulnerable plaque phenotype. PLoS One 2012;7:e43229.

36. Ozcan A, Ware K, Calomeni E, et al. 5/6 nephrectomy as a validated rat model mimicking human warfarin-related nephropathy. Am J Nephrol 2012;35:356-64.

37. Ware K, Brodsky P, Satoskar AA, et al. Warfarin-related nephropathy modeled by nephron reduction and excessive anticoagulation. J Am Soc Nephrol 2011;22:1856-62.

38. Hernandez AV, Bradley G, Khan M, et al. Rivaroxaban vs. warfarin and renal outcomes in non-valvular atrial fibrillation patients with diabetes. Eur Hear J Qual Care Clin Outcomes 2020;6:301-7.

39. Hijazi Z, Hohnloser SH, Andersson U, et al. Efficacy and safety of apixaban compared with warfarin in patients with atrial fibrillation in relation to renal function over time: insights from the ARISTOTLE randomized clinical trial. JAMA Cardiol 2016;1:451-60.

40. Yao X, Shah ND, Sangaralingham LR, Gersh BJ, Noseworthy PA. Non-vitamin $\mathrm{K}$ antagonist oral anticoagulant dosing in patients with atrial fibrillation and renal dysfunction. J Am Coll Cardiol 2017;69:2779-90.

41. Coresh J, Turin TC, Matsushita K, et al. Decline in estimated glomerular filtration rate and subsequent risk of end-stage renal disease and mortality. JAMA 2014;311:2518-31.

42. Caldeira D, Gonçalves N, Pinto FJ, Costa J, Ferreira JJ. Risk of renal failure with the non-vitamin $\mathrm{K}$ antagonist oral anticoagulants: systematic review and meta-analysis. Pharmacoepidemiol Drug Saf 2015;24:757-64.

43. Roldán V, Marín F, Fernández H, et al. Renal impairment in a 'real-life' cohort of anticoagulated patients with atrial fibrillation (implications for thromboembolism and bleeding). Am J Cardiol 2013;111:1159-64.

44. Li WH, Huang D, Chiang CE, et al. Efficacy and safety of dabigatran, rivaroxaban, and warfarin for stroke prevention in Chinese patients with atrial fibrillation: the Hong Kong atrial fibrillation project. Clin Cardiol 2017;40:222-9.

45. Chan YH, See LC, Tu HT, et al. Efficacy and safety of apixaban, dabigatran, rivaroxaban, and warfarin in Asians with nonvalvular atrial fibrillation. J Am Heart Assoc 2018;7:e008150

46. Zhang J, Tang J, Cui X, et al. Indirect comparison of novel oral anticoagulants among Asians with non-valvular atrial fibrillation in the real world setting: a network metaanalysis. BMC Cardiovasc Disord 2019;19:182.

47. Liu X, Huang M, Ye C, Zeng J, Zeng C, Ma J. The role of non-vitamin $\mathrm{K}$ antagonist oral anticoagulants in Asian patients with atrial fibrillation: a PRISMA-compliant article. Medicine 2020;99:e21025. 